\title{
Autoimmune alternating hyper- and hypo-thyroidism: a rare condition in pediatrics
}

\section{Luísa Correia Martins, Ana Rita Coutinho, Mónica Jerónimo, Joana Serra Caetano, Rita Cardoso, Isabel Dinis and Alice Mirante}

Department of Pediatric Endocrinology, Diabetes and Growth, Pediatric Unit, Coimbra Hospital and Universitary Center, 3030 Coimbra, Portugal
Correspondence should be addressed to L C Martins Email

luisacorreiamartins@ gmail.com

\section{Summary}

Alternating between hyper- and hypo-thyroidism may be explained by the simultaneous presence of both types of TSH receptor autoantibodies (TRAbs) - thyroid stimulating autoantibodies (TSAbs) and TSH blocking autoantibodies (TBAbs). It is a very rare condition, particulary in the pediatric age. The clinical state of these patients is determined by the balance between TSAbs and TBAbs and can change over time. Many mechanisms may be involved in fluctuating thyroid function: hormonal supplementation, antithyroid drugs and levels of TSAbs and TBAbs. Frequent dose adjustments are needed in order to achieve euthyroidism. A definitive therapy may be necessary to avoid switches in thyroid function and frequent need of therapeutic changes. We describe an immune-mediated case of oscillating thyroid function in a 13-year-old adolescent. After a short period of levothyroxine treatment, the patient switched to a hyperthyroid state that was only controlled by adding an antithyroid drug.

\section{Learning points:}

- Autoimmune alternating hypo- and hyper-thyroidism is a highly uncommon condition in the pediatric age.

- It may be due to the simultaneous presence of both TSAbs and TBAbs, whose activity may be estimated in vitro through bioassays.

- The clinical state of these patients is determined by the balance between TSAbs and TBAbs and can change over time.

- The management of this condition is challenging, and three therapeutic options could be considered: I-131 ablation, thyroidectomy or pharmacological treatment (single or double therapy).

- Therapeutic decisions should be taken according to clinical manifestations and thyroid function tests, independent of the bioassays results.

- A definitive treatment might be considered due to the frequent switches in thyroid function and the need for close monitoring of pharmacological treatment. A definitive treatment might be considered due to the frequent switches in thyroid function and the need for close monitoring of pharmacological treatment.

\section{Background}

Autoimmune thyroid disease is one of the most common autoimmune conditions, affecting $2-4 \%$ of women and $1 \%$ of men (1). Although its prevalence is higher in adults, it is also the most frequent etiology of acquired thyroid dysfunction in pediatrics. In this population, it is most common in girls and generally occurs in early and midpuberty (2).

Autoimmune thyroid disease encompasses an ample spectrum of thyroid disorders from which Hashimoto's

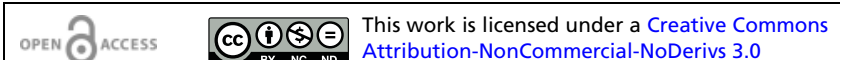
Unported License.
} 
thyroiditis and Graves' disease are the most common presentations. They represent the two ends of the autoimmune thyroid disease spectrum once they have opposite phenotypes and distinct immunologic mechanism. At first, there is T-cell infiltration of the gland leading to its destruction and clinical signs of hypothyroidism; finally, the gland is chronically stimulated by agonist antibodies of thyroid-stimulating hormone (TSH) receptor, produced by local B cells, causing hyperthyroidism.

However, there is an interrelationship between the various autoimmune thyroid disorders that could be explained by a common pathophysiological mechanism and antibody production. The thyroid gland releases antibodies against specific antigens, more frequently against thyroglobulin, thyroid peroxidase and TSH receptor. Elevated levels of antithyroglobulin and antithyroid peroxidase antibodies may not only be found in patients with autoimmune thyroid disease but also in healthy individuals, with a prevalence of $5-30 \%$ in the general population. The TSH-receptor antibodies (TRAbs) are more specific for autoimmune thyroid disease. Their prevalence in Graves' disease and autoimmune thyroiditis is $80-95 \%$ and $10-20 \%$, respectively (3).

There are two types of TRAbs: thyroid-stimulating autoantibodies (TSAbs) that cause Graves' disease and TSHblocking autoantibodies (TBAbs), competitive inhibitors of TSH binding sites without agonistic activity. TBAbs are found in a significant number (18.5\%) of adult patients with untreated Graves' disease; however, TBAbs-induced hypothryoidism is a very rare condition $(4,5)$. Some patients may have TBAbs and TSAbs sequentially and evolve from hypoto hyperthyroidism or vice versa. In very few adult patients, both antibodies may be present simultaneously, leading to a rapid oscillation in thyroid function as TBAbs or TSAbs become dominant (5). This situation is even more uncommon in pediatric population (4). The TRAbs activity may be estimated in vitro through bioassays. The more sophisticated ones involve chinese hamster ovary cells (CHO cells) transfected with the recombinant human TSHreceptor. The biological activity is deduced through cyclic adenosine $3^{\prime}, 5^{\prime}$-monophosphate (cAMP) production $(6,7)$.

Herein, we report a case of an adolescent with fluctuating thyroid function associated with elevated levels of antithyroglobulin, antithyroid peroxidase and TSH-receptor antibodies.

\section{Case presentation}

A previously healthy 13-year-old girl, referred for evaluation, presented in the clinic with a slightly increased thyroid volume, and a subclinical hyperthyroidism observed in June 2012, with no signs or symptoms (table 1). There was family history of thyroid disease.

On first evaluation, in March 2013, she presented a visible and palpable thyroid gland with no individualized nodules, no adenomegaly, and no exophthalmos. There were no other relevant findings in her physical exam. The TSH and free-thyroxine ( $\mathrm{fT}_{4}$ ) serum levels were within the normal range, but she presented with elevated levels of antithyroid peroxidase antibodies (Table 1). The thyroid ultrasound confirmed a diffuse glandular enlargement (right lobe $18 \times 16 \times 46 \mathrm{~mm}$; left lobe $16 \times 16 \times 51 \mathrm{~mm}$ ), with an heterogeneous and hypoechoic parenchymal echo pattern suggestive of thyroiditis. She was started on levoT $_{4}\left(\mathrm{LT}_{4}\right) 0.4 \mu \mathrm{g} / \mathrm{kg}$ per day to reduce goiter volume. However, after 6 months of treatment (in September 2013), she developed subclinical hyperthyroidism, and $\mathrm{LT}_{4}$ was slowly weaned off.

In June 2014, 4 months after the $\mathrm{LT}_{4}$ cessation, she gained weight rapidly ( $2.5 \mathrm{~kg}$ in 2 months), but no other signs or symptoms of hypothyroidism were reported and there was no increase in goiter volume. At this time, TSH was $46 \mu \mathrm{U} / \mathrm{ml} \mathrm{fT}_{4}$ was $0.5 \mathrm{ng} / \mathrm{dl}$ and there was an elevation in the TRAbs adding to elevated TPO (Table 1). After 3 days, a repeat TSH measurement showed a spontaneous decrease in TSH to $14.2 \mu \mathrm{U} / \mathrm{ml}$, without any treatment, and the scintigraphy showed a homogeneously increased iodine uptake, suggestive of Graves' disease. She was again started on $\mathrm{LT}_{4}$ at $0.73 \mu \mathrm{g} / \mathrm{kg}$ per day. On follow-up after 2 months of $\mathrm{LT}_{4}$ treatment (in August 2014), she was noted to have a decrease in TSH and an increase in $\mathrm{fT}_{4}$, with no signs of hyperthyroidism apart from weight loss (Table 1). She maintained treatment with $\mathrm{LT}_{4}$. Methimazol was started at a dose of $0.15 \mathrm{mg} / \mathrm{kg}$ per day and increased after 2 months to $0.20 \mathrm{mg} / \mathrm{kg}$ per day due to laboratory evidence of persistent hyperthyroidism. Thyroid function was controlled with this double therapeutic regimen for a period of time, but after 6 months she again developed hyperthyroidism that demanded another adjustment, with a decrease in $\mathrm{LT}_{4}$ to $0.37 \mathrm{mg} / \mathrm{kg}$ and increase in methimazol to $0.23 \mathrm{mg} / \mathrm{kg}$ daily (in July 2015). At this point, on suspicion of the coexistence of TBAbs and TSAbs as a cause of these rapid oscillations in thyroid function, a sample of serum was sent to Hospices Civils de Lyon to perform a bioassay to measure TSAbs and TBAbs activity (the method is described in Table 2). The serum level of TSH was $<0.004 \mu \mathrm{U} / \mathrm{ml} \mathrm{fT}_{4}$ was $3.1 \mathrm{ng} / \mathrm{dl}$ and TRAbs were $20 \mathrm{UI} / \mathrm{l}$. A moderate TSAbs activity was detected (203\%), but there was no evidence of blocking activity. 


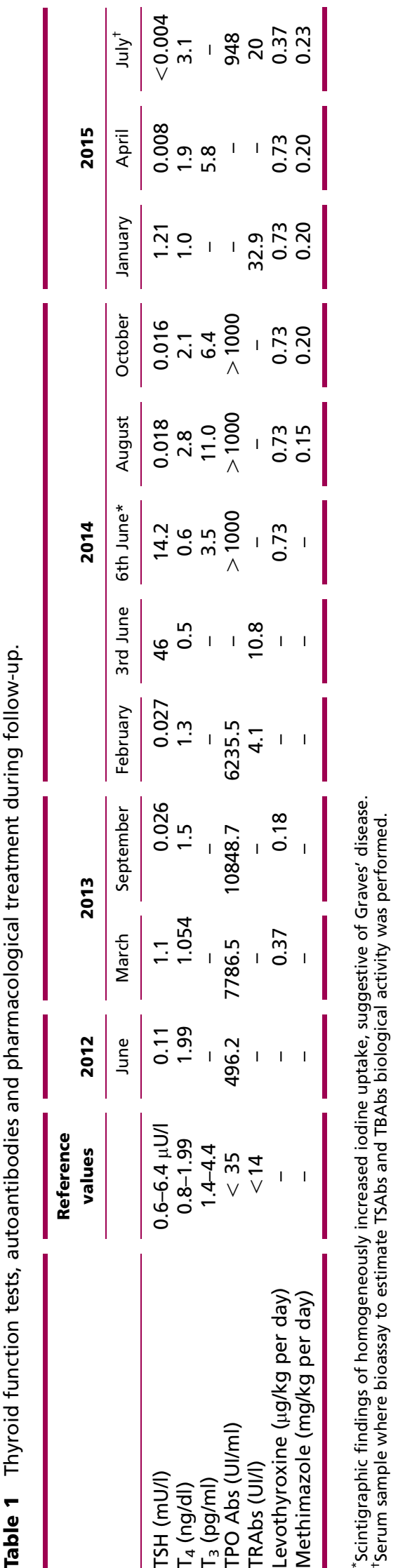

\section{Discussion}

In this clinical case, there is an alternation between hyperand hypothyroidism associated with elevated levels of antithyroglobulin, antithyroid peroxidase and TRAbs, with ultrasonographic and scintigraphic findings compatible both with thyroiditis and Graves' disease, respectively. A hyperthyroid state was evident at the early onset in June 2012, with spontaneous remission, and after the beginning of $\mathrm{LT}_{4}$ therapy in March 2013, to reduce goiter volume. Effectiveness of $\mathrm{LT}_{4}$ treatment in reducing goiter volume in euthyroid children and adolescents with autoimmune thyroid disease is not clear yet, although recent data supports its use $(8,9)$. However, due to the possibility of $\mathrm{LT}_{4}$ overtreatment as a cause of hyperthyroidism noticed in September 2013, $\mathrm{LT}_{4}$ was slowly weaned off until its cessation. After a period without treatment, she unexpectedly developed hypothyroidism (in June 2014), associated with elevated levels of TRAbs. Notice also that without any medication, TSH levels decreased significantly within 3 days and that at this point scintigraphic findings were compatible with Graves' disease. $\mathrm{LT}_{4}$ was restarted and, once again, a switch to hyperthyroidism was noted right after the beginning of $\mathrm{LT}_{4}$ that was only reverted with the addition of methimazol. Further therapeutic adjustments were needed, due to thyroid function fluctuation over time.

This type of oscillation in thyroid function is extremely rare, especially in the pediatric age, and is only described in a few case reports in literature $(4,5)$. Takasu \& Matsushita (10) admitted that this fluctuation in thyroid function can be explained by the simultaneous presence of TSAbs and TBAbs in the sera of the patient. The balance between both antibodies determines whether a patient has hypo- or hyperthyroidism (10). McLachlan \& Rapoport (5) advocate that a number of mechanisms may be behind the pendulum swinging from TBAbs and TSAbs: i) treatment with $\mathrm{LT}_{4}$; ii) treatment with antithyroid drugs and iii) inherent properties of TSAbs and TBAbs. In some patients, $\mathrm{LT}_{4}$ therapy may be associated with an increase in autoantibody responses due to the effect of $\mathrm{LT}_{4}$ in innate and adaptive immune responses (5). This may explain the reason why our patient developed hyperthyroidism in a short period after she was started on $\mathrm{LT}_{4}$. On the other hand, treatment with antithyroid drugs, in particular methimazole, may lead to a decrease in the levels of thyroid autoantibodies by a direct mechanism of inhibition of its synthesis (5). In this case, no blocking activity was detected during a hyperthyroid state, 1 year after double therapy, which may be due to the influence methimazole in immune responses and antibody 
Table 2 Bioassay descriptions for measurement of TRAbs biological activity $(6,7)$.

Bioassays location

Procedure

TRAbs biological activity

Variability between assays

\section{Biology Department of the Hospices Civils de Lyon Sud University Hospital}

The CHO cells (strain JP-26) are transfected with the recombinant human TSH receptor

The CHO JP-26 cells are seeded into 96-well plates (50 000 cells/well), cultured in Ham's F12 medium, containing $5 \%$ calf serum, and used for TSAb and TBAb bioassays $24 \mathrm{~h}$ after seeding

The $\mathrm{CHO}$ cells were exposed for $2 \mathrm{~h}$ to $4 \mathrm{ml}$ of test serum or control serum in $196 \mathrm{ml}$ with $10 \mathrm{mM}$ HEPES, $0.25 \mathrm{mmol} / \mathrm{l}$ isobutylmethylxanthine, and $0.75 \%$ bovine seric albumin, $\mathrm{pH} 7.4$

For the TBAb bioassay, the hypotonic medium was supplemented with bovine TSH $(0.1 \mathrm{mIU} / \mathrm{ml})$

After incubation, CAMP released from the cells was measured with a commercial RIA Kit (RIA cAMP, IMMUNOTECH, a Beckman Coulter Company, Marseille, France) according to the manufacturer's instructions

Pooled TRAb-negative sera (normal sera) were used to measure CAMP basal production, pooled TSAb-positive sera were used as positive controls in TSAb assay and pooled TBAb-positive sera as positive controls in TBAb assay

TSAb activity was expressed as a percentage of CAMP basal production

TSAb activities ranging from:

$140-200 \%$ were considered as weak

$200-400 \%$ were considered as moderate

$\geq 400 \%$ were considered as strong

TBAb activity was calculated and expressed as follows: $1-(a / b) \times 100$, where:

(a) is the CAMP generated in the presence of the patient's sample and bovine TSH

(b) is the cAMP generated in the presence of normal sera and bovine TSH

TBAb activities ranging from:

$10-20 \%$ were considered as weak

$20-40 \%$ were considered as moderate

$\geq 40 \%$ were considered as strong

The assays were run in triplicate, and results are expressed as the mean of the three data

TSAb activity: $8.6 \%$

TBA activity: $7.1 \%$ production, leading to a decrease of TBAbs to undetectable levels. Another important mechanism that might be involved is the TSAbs' and TRAbs' affinities for the TSH receptor. Clinical status of a patient in a particular point of time may reflect not only the concentration of both antibodies, but also their different affinities for the receptor, and the antibodies with higher concentrations and affinity will dictate the clinical presentation (5).

The bioassays to determine biological activity of TSAbs and TBAbs may be very useful to clarify specific situations, when clinical manifestations suggest the presence of TBAbs. However, once they are not widely available, and there are various mechanisms that may influence TBAbs and TSAbs biologic activity over time, their clinical utility may be limited in situations of alternating thyroid function. McLachlan \& Rapoport (5) emphasize that therapeutic decisions should be taken according to clinical manifestations and thyroid function tests, independent of the bioassays results.

The management of this condition is challenging, and three therapeutic options could be considered: I-131 ablation, thyroidectomy or pharmacological treatment (single or double therapy). This last option requires frequent thyroid function monitoring to titrate drugs. Mathew \& Moore (4) reported two similar cases, in a 5- and an 8-year-old girl in whom an appropriate single drug regimen failed to control thyroid function. In both cases, and considering their age, patients underwent a total thyroidectomy (4). In this case, double therapy briefly controlled thyroid function, with frequent dose adjustment needed. Definitive treatment with I-131 is being considered as a suitable option for our patient to avoid the switches in thyroid function.

Another unexpected fact in this clinical case is the scintigraphic findings of a homogeneously increased iodine uptake, suggesting Graves' disease, during a hypothyroidism state (in June 2014). There are no data in literature about scintigraphic findings in patients with an oscillation of thyroid function. One possible explanation is that the scintigraphy was done in a transition phase between hypo- and hyper-thyroidism, once TSH decreased significantly within 3 days and TRAbs were elevated. This fact may lead to an overstimulation of the thyroid that could be responsible for the homogeneously increased iodine uptake in scintigraphy. Further studies are needed to confirm this hypothesis and to better understand this phenomenon.

\section{Conclusion}

Autoimmune alternating hypo- and hyper-thyroidism is a highly uncommon challenging condition, particularly in 
the pediatric age, and is due to the simultaneous presence of both TSAbs and TBAbs. The clinical status of the patient may reflect the balance between these autoantibodies and can be influenced by their concentration, affinities and therapeutic interventions ( $\mathrm{LT}_{4}$ and antithyroid drugs). The bioassays that determine biological activity of TSAbs and TBAbs may be useful; however, their clinical utility is limited in situations of alternating thyroid function. Therapeutic decisions should be taken according to clinical manifestations and thyroid function tests, independent of the bioassays results. Adding an antithyroid drug to $\mathrm{LT}_{4}$ treatment might be necessary to achieve better thyroid function control. A definitive treatment should be considered due to the frequent switches in thyroid function and the need for close monitoring of pharmacological treatment.

\section{Declaration of interest}

The authors declare that there is no conflict of interest that could be perceived as prejudicing the impartiality of the research reported.

\section{Funding}

This research did not receive any specific grant from any funding agency in the public, commercial or not-for-profit sector.

\section{Patient consent}

A written informed consent was obtained from the patients' guardians for publication of the submitted article.

\section{Author contribution statement}

L C Martins, A R Coustinho and M Jerónimo were responsible for patient care, bibliographic research and paper writing; J S Caetano, R Cardoso, I Dinis and A Mirante were responsible for patient care and paper revision.

\section{Acknowledgements}

We would like to thank Dr. Karim Chikh (Hospices Civils de Lyon, Centre Hospitalier Lyon Sud, Centre de Biologie, Service de Biochimie, France and
Université de Lyon, Université Claude Bernard Lyon 1, Faculté de Pharmacie, 69008 Lyon, France) for the measurement of TSAbs and TBAbs activity.

\section{References}

1 Canaris GJ, Manowitz NR, Mayor GM \& Ridgway EC 2000 The Colorado thyroid disease prevalence study. Archives of Internal Medicine 160 526-534. (doi:10.1001/archinte.160.4.526)

2 Cappa M, Bizzarri C \& Crea F 2011 Autoimmune thyroid diseases in children. Journal of Thyroid Research. Article ID 675703. (doi:10.4061/ 2011/675703)

3 Jorge Z, Nobre EL, Santana A \& Castro JJ 2005 Doença auto-imune da tiroideia. Acta Médica Portuguesa 18 88-92.

4 Mathew RP \& Moore DJ 2011 Autoimmune Alternating Hypo- and Hyperthyroidism in Children. Clinical Pediatrics 50 1040-1044. (doi:10.1177/0009922811412583)

5 McLachlan SM \& Rapoport B 2013 Thyrotropin-blocking autoantibodies and thyroid-stimulating autoantibodies: potential mechanisms involved in the pendulum swinging from hypothyroidism to hyperthyroidism or vice versa. Thyroid 23 14-24. (doi:10.1089/thy. 2012.0374)

6 Abeillon-du Payrat J, Chikh K, Bossard N, Bretones P, Gaucherand P, Claris O, Charrié A, Raverot V, Orgiazzi J, Borson-Chazot F et al. 2014 Predictive value of maternal second-generation thyroid-binding inhibitory immunoglobulin assay for neonatal autoimmune hyperthyroidism. European Journal of Endocrinology 171 451-460. (doi:10.1530/EJE-14-0254)

7 Marques P, Chikh K, Charrié A, Pina R, Bugalho MJ \& Lopes L 2015 Hypothyroidism associated to TSH hormone-receptor autoantibodies with blocking activity assessed in vitro. Acta Médica Portuguesa 28 663-666.

8 Svensson J, Ericsson UB, Nilsson P, Olsson C, Jonsson B, Lindberg B \& Ivarsson SA 2006 Levothyroxine treatment reduces thyroid size in children and adolescents with chronic autoimmune thyroiditis. Journal of Clinical Endocrinology and Metabolism 91 1729-1734. (doi:10.1210/jc.2005-2400)

9 Scarpa V, Kousta E, Tertipi A, Vakaki M, Fotinou A, Petrou V, Hadjiathanasiou C et al. 2010 Treatment with thyroxine reduces thyroid volume in euthyroid children and adolescents with chronic autoimmune thyroiditis. Hormone Research in Paediatrics 73 61-67. (doi:10.1159/000271917)

10 Takasu N \& Matsushita M 2012 Changes of TSH-stimulation blocking antibody (TSBAb) and thyroid stimulating antibody (TSAb) over 10 years in 34 TSBAb-positive patients with hypothyroidism and in 98 TSAb-positive Graves' patients with hyperthyroidism: reevaluation of TSBAb and TSAb in TSH-receptor-antibody (TRAb)-positive patients. Journal of Thyroid Research. Article ID 182176. (doi:10.1155/2012/182176)

Received in final form 12 January 2016

Accepted 9 February 2016 